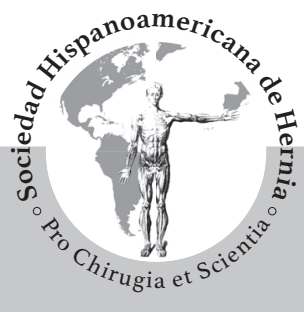

\title{
El cincuentenario de la «transposición con el saco herniario» de Lázaro da Silva para reconstrucción de la pared abdominal ventral
}

\author{
The 50th anniversary of Lázaro da Silva's technique \\ "the hernial sac transposition" for ventral abdominal \\ wall reconstruction
}

\section{Renato Miranda de Melo}

Cirujano General, responsable de los servicios de Hernias Complejas en Santa Casa de Misericordia de Goiânia y Hospital General de Goiânia (Goiânia, Goiás, Brasil). Profesor Asociado (doctor). Departamento de Cirugía. Facultad de Medicina de la Universidad Federal de Goiás (FM-UFG) e de la Pontificia Universidad Católica de Goiás (PUC). Goiânia, Goiás (Brasil). Miembro Titular del Colegio Brasileño de Cirujanos (TCBC), de la Sociedad Brasileña de Hernia y Pared Abdominal (SBH) y de la Sociedad Hispanoamericana de Hernia (SoHAH)

Recibido: 04-01-2018

Aceptado: 02-02-2018

\section{Palabras clave:}

Pared abdominal, hernia incisional, hernia ventral, herniorrafia.

\section{Resumen}

En 1968, en el interior de Brasil o, más específicamente, en Belo Horizonte, capital del estado de Minas Gerais, frente a las dificultades de acceso a las raras y costosas mallas de polipropileno, nacía una propuesta para corregir las hernias incisionales medianas, fundamentada en incisiones de descarga en las vainas de los músculos rectos del abdomen y en la utilización del propio saco herniario. En este momento el procedimiento era hecho por Alcino Lázaro da Silva, un cirujano general contumaz y entonces profesor de la Facultad de Medicina local, que no se conformaba con descartar el saco herniario, en su opinión una estructura vital y resistente, que podría ser útil en la corrección de aquellas hernias. En los años anteriores, fue divisando la técnica que denomino «transposición peritoneo-aponeurótica longitudinal bilateral»o, más brevemente, «transposición con el saco herniario» (TSH), su mayor diferencial. Aquí se relata la historia que lo llevó a elaborar su propuesta, la descripciónn textual e iconográfica del procedimiento y sus principios orientadores, además del razonamiento que fundamenta los resultados favorables obtenidos con la adopción de la técnica. En este año de 2018, se conmemora el cincuentenario de la TSH, una de las alternativas que el cirujano general podrá disponer, para la reconstrucción de la pared ventral del abdomen, sin el uso de prótesis sintéticas.
Key words:

Abdominal wall, incisional hernia, ventral hernia, herniorrhaphy.

\begin{abstract}
Fifty years ago, in Belo Horizonte, Minas Gerais, Brazil, a general surgeon and teacher, Alcino Lázaro da Silva, performed for the first time a midline incisional hernia repair using the hernial sac transposition (HST) technique. Based in long relaxing incisions all the way from angle to angle of the rectus sheath, posteriorly on the left side and anteriorly on the right side, each half of the cut hernial sac is transposed and sutured to the lateral opposite wound edge in the sheaths. Between them, both medial edges are sutured together, medializing again the rectus muscles and restoring a new linea alba. This is a true ventral abdominal wall reconstruction showing a 7\%-18\% long-term recurrence rate for these known catastrophic cases. This ingenious but very simple procedure devised by Prof. Lázaro da Silva, half a century ago, provides a solid ventral repair without meshes. For those reasons its $50^{\text {th }}$ Anniversary should be celebrated and it must have more and more widespread usage worldwide.
\end{abstract}

* Autor para correspondencia: Renato Miranda de Melo. Servicio Hernias Complejas. Santa Casa de Goiâna y Hospital General de Goiâna. Rua 5, $223 / 801$ - Setor Oeste 74115-060 - Goiânia, Goiás (Brasil)

Correo electrónico: dr.renatomelo@gmail.com

Miranda de Melo R. El cincuentenario de la «transposición con el saco herniario» de Lázaro da Silva para reconstrucción de la pared abdominal ventral. Rev Hispanoam Hernia. 2018;6(3):156-162 


\section{La historia}

Inconformado con el descarte rutinario del saco herniario, durante la reparación quirúrgica de las eventraciones, el profesor Alcino Lázaro da Silva (fig. 1), entonces vinculado a la Facultad de Medicina de la Universidad de Minas Gerais, vislumbraba ante aquella estructura una buena y tal vez la única salida para corregir esas afecciones. En el Brasil de principios de la década de 1960, las prótesis de la pared abdominal eran escasas y muy costosas. Metódico y perseverante, insistía en la elaboración de un arreglo músculo-aponeurótico, que pudiera corregir el defecto parietal, recomponiendo la línea alba, sin provocar tensión excesiva en las líneas de sutura ni en el compartimiento abdominal. Con eso alejaba la necrosis isquémica de los tejidos implicados, y la recidiva inevitable, pero también el grave síndrome compartimental. Con solo hilos de seda o algodón, formadores por excelencia de los indeseables granulomas supurativos, vía en aquella prerrogativa - reparación con poca tensión-la posibilidad de utilizar el catgut en las suturas, evitando las secuelas de los materiales multifilamento y no absorbibles.

La alternativa era hacer incisiones relajadoras (de descarga), ya practicadas, o emplear el saco herniario, o ambos. ¿Por qué no? El saco era un tejido que estaba siempre allí, disponible, exuberante, autólogo, en la temperatura corporal y listo para usar. Se decidió entonces a adoptar las dos opciones tácticas, pero eso solo pasaba en su imaginación, sin definición todavía del pretendido reordenamiento de la pared, que vendría insidiosamente, caso por caso, sin atropellar los pasos. El cambio definitivo para Belo Horizonte a la capital del estado, trae mayor número de pacientes y su total dedicación a las lides universitarias y mayor atención a la familia. $\mathrm{Su}$ experiencia se fue acumulando y la manera de corregir las hernias incisionales se fue haciendo más definida en la mente del profesor Alcino, que pasó a establecer la mejor indicación para su propuesta: serían las eventraciones medianas o paramedianas, con anillo elíptico craneocaudal, único, y con el saco herniario también único y amplio. ¡Cuanto más grande, mejor!

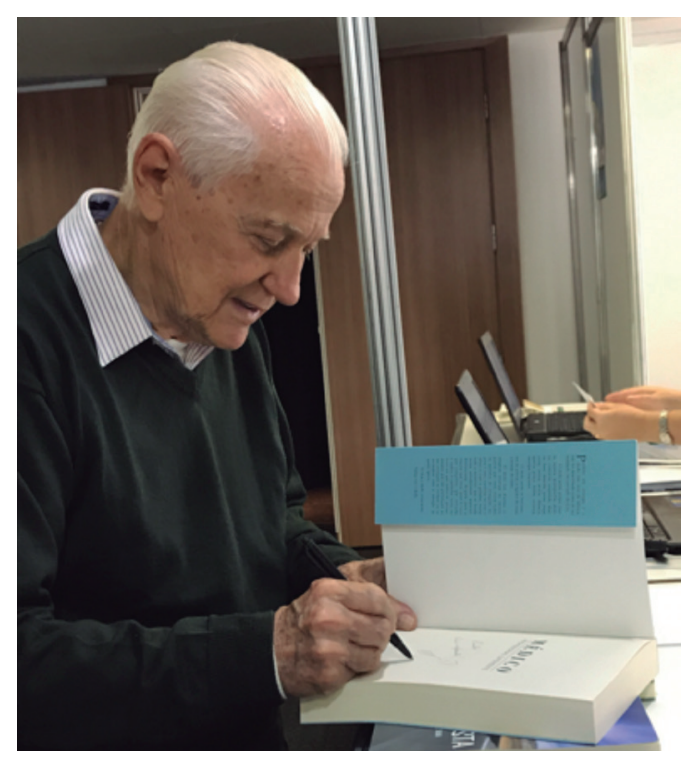

Figura 1. Profesor Alcino Lázaro da Silva (archivo privado).
En el año siguiente, operando y reevaluando las posibilidades, revisando la anatomía local, ensayando aquí y allí, fue componiendo la técnica, hasta que llegó al día 9 de noviembre de 1968, cuando se presentó un señor de 72 años, labrador, con hernia incisional mediana supraumbilical poscolecistectomía. La descripción sumaria y el esbozo que constan en el prontuario del paciente, facilitado por el propio profesor Alcino, apuntan al arreglo de lo que él mismo llamó, más tarde, «transposición peritoneo-aponeurótica longitudinal bilateral» (TRANSPALB), perpetrada con catgut como siempre. Incluso aquí, el relato más antiguo del arreglo más cercano de lo que es, hoy, la transposición, difiere en el modo en que reaproximó los músculos rectos en la línea media. Este detalle, más tarde, sufrió un pequeño perfeccionamiento que, de hecho, vendría a restablecer una línea alba aponeurótica e individualizada. Por lo tanto, parece lícito considerar esta la fecha inaugural de la TRANSPALB, o más simplemente transposición con el saco herniario (TSH), por retratar la concepción original y la esencia de la técnica idealizada, hace medio siglo, por el profesor Alcino Lázaro da Silva.

En 1971, se publicó la primera serie de 15 pacientes así operados (el más antiguo de ellos tres años antes), bajo el título «Plástica con el saco herniario en la corrección de las hernias incisionales», con dibujos hechos a mano libre por el autor, en la extinta revista El Hospital, en su volumen 79, pp. 123 a 134 (fig. 2). Pasados otros tres años, inaugurando la Revista del Colegio Brasileño de Cirujanos, el autor publicó su serie acumulada con 55 pacientes, habiendo extendido las indicaciones también para las hernias paramedianas y las diástasis mayores (Revista del Colegio Brasileño de Cirujanos 1974;1:113-6). Cinco años después, con la experiencia ya sedimentada, la técnica fue presentada a la

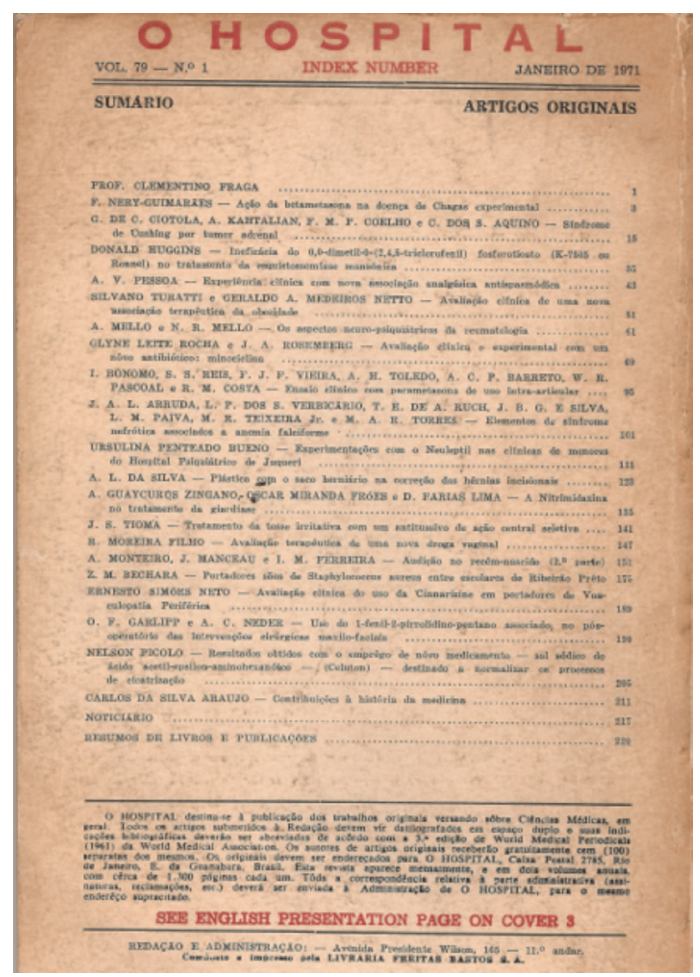

Figura 2. Resumen de la revista El Hospital (primera comunicación de la transposición con el saco herniario). 
comunidad científica internacional, en la también extinta Surgery, Gynecology and Obstetrics (continuada como Journal of the American College of Surgeons), habiendo recibido una acogida tímida, sobre todo en Argentina, España, Francia y Gran Bretaña. Hace 10 años, una revisión de literatura reconoció la técnica brasileña como una de las alternativas para la corrección de las hernias incisionales, sin el uso de malla, sucediendo a la separación de componentes, propuesta por Ramírez en $1990^{1,2}$.

Otros estudios sobrevinieron, la mayoría académicos, a nivel de posgrado, tanto experimentales como series de casos, evaluando complicaciones posoperatorias e índices de recidiva. Uno de ellos empleaba la TSH como estrategia en el cierre precoz de pacientes con «abdomen abierto» ${ }^{3}$. En el año 2004, el propio profesor Alcino publicó su casuística personal de 52 pacientes, con seguimiento que varía de 6 meses a 22 años (promedio de 4 años y 10 meses), presentando una tasa de recidiva del orden del $7.69 \%(4 / 52)^{4}$. A pesar de ser más una serie de casos, con amplia variación en el seguimiento posoperatorio, el resultado fue sorprendente, al menos en manos de su idealizador, casi la mitad a la del uso sistemático de mallas, apuntada en un reciente artículo de revisión ${ }^{5}$.

\section{La técnica}

\section{Indicaciones y preparación del paciente}

La mejor indicación para emplear la TSH es en la corrección de hernias incisionales medianas, con defecto único y elíptico (longitud mayor que la anchura), supra y/o infra-umbilical, y saco herniario grande y espeso. Para ello, es recomendable espaciar cerca de seis a doce meses de la operación inicial, que generó la eventración, para que pueda haber su debida «aponeurotización». Es otra posibilidad para el tratamiento de las grandes diástasis, con distancia interrretal $>7.5 \mathrm{~cm}(3 \times$ la anchura media de la línea alba supra y meso-umbilical), acompañadas o no de hernias epigástrica y/o umbilical. La razón última para indicar esta técnica no puede ser la indisponibilidad de mallas, sino por tratarse de un procedimiento cuyo resultado se equipara a las reparaciones protésicas, pero sin el uso de esos materiales. Por eso el cirujano deberá atenerse a los criterios de mejor indicación de la técnica, para obtener los mejores resultados posibles. No se admite la panacea en el universo de las hernias ni de cualquier otra afección.

Aunque no sea un procedimiento que promueve hipertensión excesiva en las líneas de sutura o en la cavidad abdominal, son fundamentales la evaluación y la preparación adecuadas de la parte respiratoria, con fisioterapia, obligatoriamente, y medicamentos, si es necesario, además de los cuidados sistémicos y locales, sobre todo con la piel, que a menudo presenta cicatrices, pliegues y sobras (abdomen en delantal), todas muy frecuentes en estos casos. En este contexto, vale resaltar que la dermolipectomía se impone, como vía de acceso, y no por razones meramente estéticas. El condicionamiento físico del paciente, iniciado antes del procedimiento, debe extenderse en el posoperatorio, a fin de evitar complicaciones potencialmente graves como el tromboembolismo venoso y la atelectasia. Al lado de una rigurosa y completa evaluación clínica, exámenes de imagen, del tipo tomografía computarizada, auxilian en la apreciación de las dimensiones del defecto herniario, de su contenido y del remanente parietal, con finalidad diagnóstica y de planificación quirúrgica. Algunas veces, se hace necesaria también la expansión anticipada del continente abdominal, sea con el neumoperitoneo progresivo o incluso con la toxina botulínica del tipo $\mathrm{A}$, o ambos, añadiendo todas las comodidades que tales medidas prestan a cualquier técnica de reparación de la pared abdominal.

\section{Anestesia y medidas preliminares}

La asociación de bloqueo espinal, con o sin la colocación de catéter en el espacio extradural, tiene la finalidad de promover analgesia posoperatoria prolongada, evitando disturbios cardiorrespiratorios, entre otros y anestesia general, que favorece el manejo visceroparietal, es con certeza más seguro y cómodo para todos, paciente y equipo.

El campo operatorio deberá ser preparado de la manera habitual, con antisepsia rigurosa, pero la salvedad de que la aplicación de campos adhesivos sobre el saco herniario no es recomendable, pues el volumen herniado, la redundancia de piel y las reentradas cicatriciales hacen la superficie bastante irregular, impidiendo el ajuste adecuado de ese material sobre la pared. El cateterismo vesical es facultativo, pues el procedimiento total tiene una duración media de dos a tres horas, y así se evitan los riesgos de contaminación de la vía urinaria. De la misma forma es la preparación de colon, salvo cuando fuertemente indicativo de envolvimiento de ese segmento en el contenido herniado.

La profilaxis antimicrobiana se realiza con una cefalosporina de primera generación (cefazolina), en dosis única, administrada por vía intravenosa en la inducción anestésica. En este sentido, se deben adoptar todas las medidas de asepsia, aunque se trate de una cirugía potencialmente contaminada, además del manejo delicado con los instrumentos, procurando minimizar daños adicionales a los tejidos.

La incisión de piel deberá comprender la remoción total, siempre que sea posible, de la cicatriz cutánea, con objetivo no solo estético, pero principalmente para que el cirujano tenga acceso inmediato al tejido subcutáneo sano, fuera de la zona cicatricial en este estrato, que dificulta la disección del saco herniario (SH), localizado justo debajo de la piel y, en la mayoría de las veces, firmemente adherido o ya fundido a ella.

La disección prosigue, entonces, en sentido centrífugo, buscando siempre tangenciar el $\mathrm{SH}$, que deberá permanecer íntegro y con el mínimo de tejido graso adherido a él, lo que significa preservar el espesor y la nutrición de los retazos dermograsos. A medida que se lateraliza esa misma disección, bilateralmente, deberán ser identificados y preservados, en la medida de lo posible, los haces vasculonerviosos que alcanzan esos retazos, lo que hace reducir las áreas de isquemia $\mathrm{y}$, consecuentemente, la ocurrencia de necrosis y de infección del sitio quirúrgico. Al final, se expone completamente el defecto parietal, el SH redundante y el plano aponeurótico adyacente, representado por la lámina anterior de la vaina de los músculos rectos del abdomen (MRA), en su porción más medial, a cada lado, alrededor de $3 \mathrm{~cm}$ a la izquierda y de 5 $\mathrm{cm}$ a la derecha, y no más que eso, de lo contrario, se aumenta mucho la incidencia de seroma. De la misma forma, los ángulos craneal y caudal del anillo herniario deben ser ampliamente expuestos. Las compresas se colocan en ese espacio, recién disecado, para protegerlo y mantenerlo exangüe (fig. 3 ). 


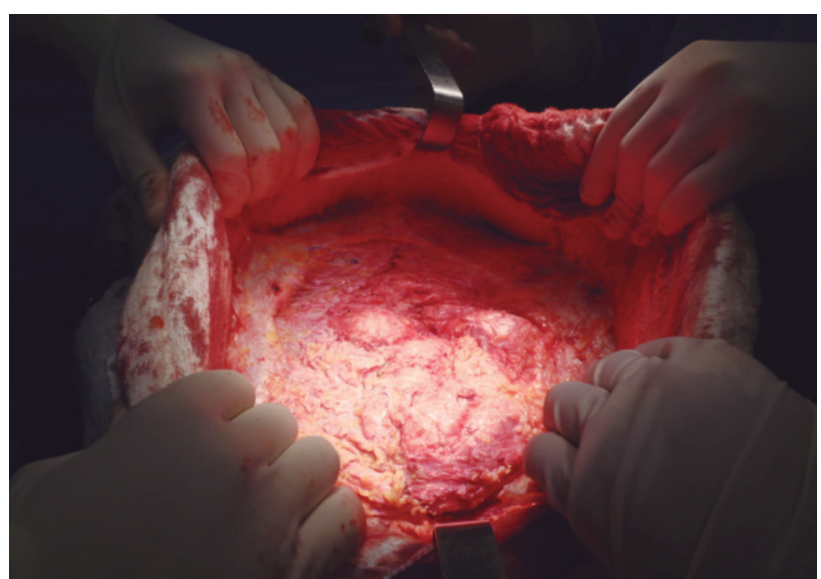

Figura 3. Defecto parietal con el saco herniario todavía cerrado.

\section{Las incisiones relajantes y la preparación de los folletos}

El SH se abre longitudinalmente, en la línea media, con el debido cuidado para no lacerar inadvertidamente tirantes intestinales o incluso el omento, pues quedan adheridos a su cúpula, a veces de modo muy firme (fig. 4). Es preferible dejar pequeños fragmentos de saco en las asas, que lo contrario. La lisis completa de adherencias será obligatoria solo del lado que recibirá la incisión relajadora, posteriormente, en general a la izquierda, por razones meramente ergonómicas. Se confecciona muy cerca del borde medial del músculo $(2 \mathrm{~cm})$, tiene un tramo curvilíneo, de un ángulo a otro, pudiendo sobrepasarlos (fig. 5). Deshacer el ligamento falciforme del hígado, de ese mismo lado, es fundamental para exponer adecuadamente la lámina posterior de la vaina, en su porción supraumbilical. El úraco y la grasa supravesical, a su vez, también son rebatidos para la extensión caudal de esa incisión. Se expone la cara profunda del MRA y los vasos epigástricos inferiores, que se preservan, definiendo los tres folletos posteriores a la izquierda: el lateral, el medial y el borde medial libre del SH.

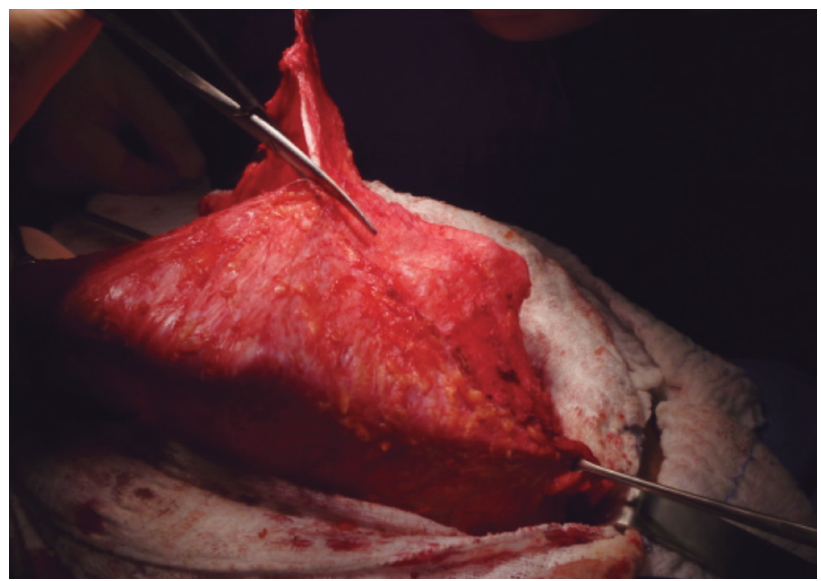

Figura 4. Apertura longitudinal del saco herniario.

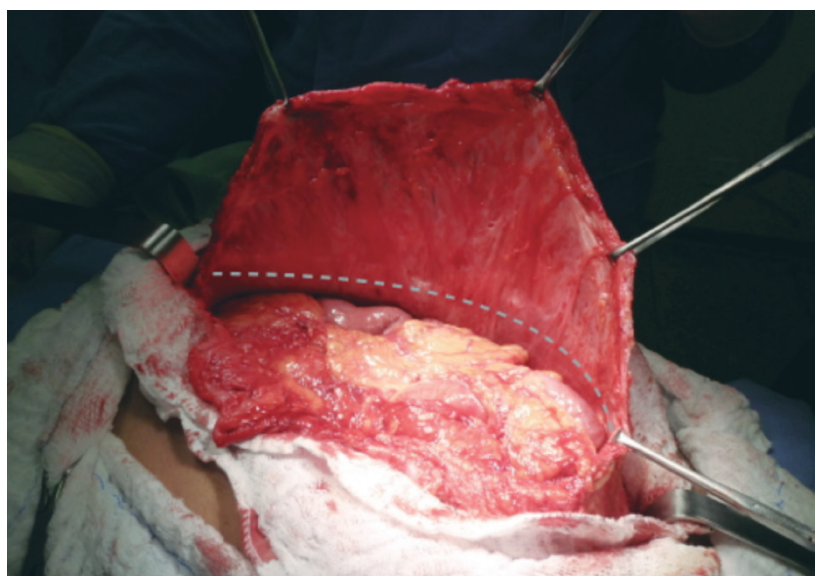

Figura 5. Cara profunda de la pared abdominal a la izquierda (la línea discontinua demarca el lugar de la incisión en la lámina posterior de la vaina del músculo rectoabdominal).

En el lado opuesto, la lámina anterior de la vaina es hendida, de modo semejante, exponiendo el vientre y las intersecciones tendíneas del MRA. Aquí se delimitan los tres folletos anteriores a la derecha: el lateral, el medial y el borde medial libre del SH (fig. 6).

En ese momento, la hemostasia es rigurosamente asegurada, así como las compresas son conferidas. Es recomendable cubrir las vísceras con el omento mayor, para evitar su implicación inadvertida durante la síntesis de la pared.

\section{La transposición con el saco herniario}

A partir de aquí, comienza la reconstrucción de la pared, que corregirá el defecto herniario o la gran diástasis, al medializar los MRA nuevamente y restablecer la línea alba con tejido aponeurótico. Para ello se realizan tres planos de sutura, en los que se pueden utilizar hilos absorbibles sintéticos, preferentemente los monofilamentos de larga duración, tales como la polidioxanona o el polibutirato, de calibre 0 o incluso 2-0, en chuleio simple (no festoneado).

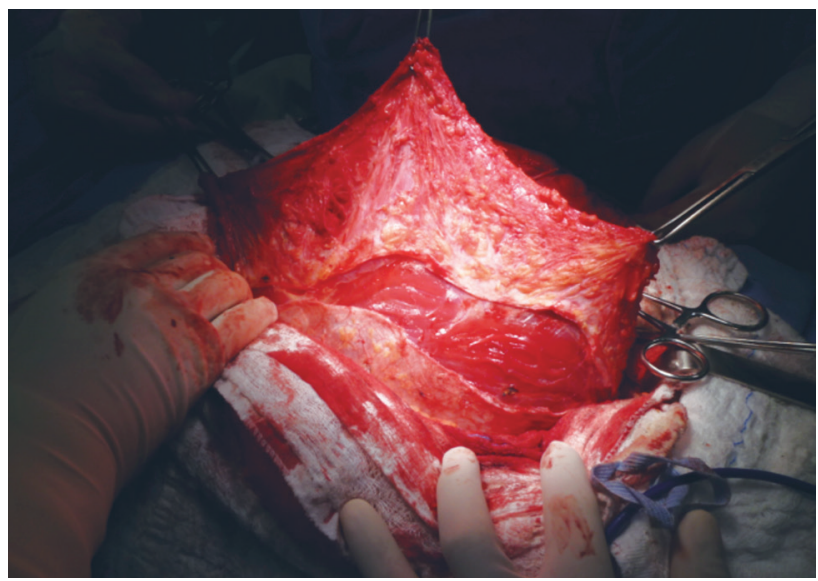

Figura 6. Folletos anteriores a la derecha (lateral, medial y borde libre de la mitad derecha del saco herniario). 
- 1. ${ }^{\text {er }}$ plano: el borde libre de la mitad derecha del SH (hemi$\mathrm{SH})$ se sutura al folleto posterior lateral izquierdo. Aquí la cavidad peritoneal es cerrada completamente y el MRA izquierdo es cubierto nuevamente, dando inicio a la recomposición de su vaina (fig. 7A).

- 2. ${ }^{\mathrm{o}}$ plano: los folletos medianos, anterior derecho y posterior izquierdo, son suturados, reaproximando los MRA y rehaciendo la línea alba. Aquí también se completa la recomposición de la vaina del MRA izquierdo y se inicia la del músculo derecho (fig. 7B).
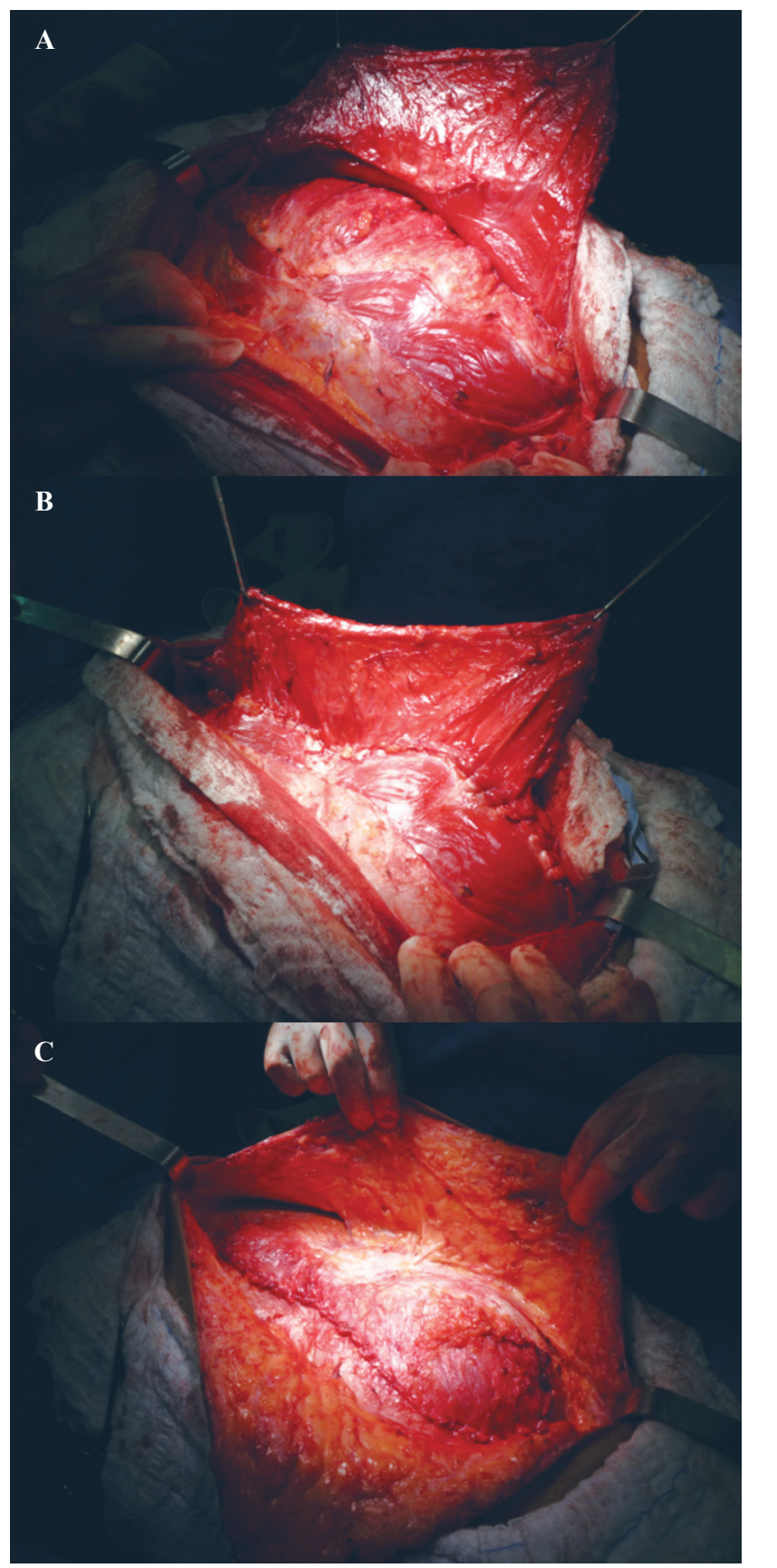

Figura 7. Transposición con el saco herniario, mostrando completado cada uno de los tres planos de sutura (A, B, C).
- 3. er $^{\text {p }}$ lano: el borde libre de la mitad izquierda del SH (hemi$\mathrm{SH})$ se sutura al folleto anterior lateral derecho. Aquí se completa la recomposición de la vaina del MRA derecho (fig. 7C).

Este avance musculoaponeurótico bilateral solo es posible realizarlo sin tensión excesiva gracias a las dos incisiones relajadoras realizadas. La TSH no altera la función pulmonar en el postoperatorio $^{6,7}$. Tal hecho puede ser constatado también durante el procedimiento, en pacientes intubados, obviamente, monitoreando la presión de meseta traqueal $\left(\mathrm{cmH}_{2} \mathrm{O}\right)$, que se mantiene inalterada $\mathrm{u}$ oscila poco (hasta un $20 \%$ de los niveles basales), al final de cada plano suturado. La validez de estas medidas dependerá del plano anestésico y de la relajación neuromuscular adecuada.

Otro hecho, observado en la confección del 2. ${ }^{\circ}$ plano es el desgarrador aunque parcial del MRA derecho, en virtud de las intersecciones tendidas que lo fijan a la lámina anterior de su vaina, la cual es hendida de este lado. Es reversible, en la medida en que la mitad izquierda del SH lo recubrirá, estabilizándolo en su nueva tienda y permitiendo que la actividad física posoperatoria termine por restablecer su integridad y función.

La hemostasia del tejido subcutáneo es también revisada, para que sea entonces reasentado en el lecho aponeurótico y ahí fijado con varios puntos de hilo absorbible, cada $4 \mathrm{~cm}$ o $5 \mathrm{~cm}$, a la manera de Baroudi, reduciendo al máximo el espacio vacío, en esa interfaz, lo que previene la acumulación de seromas ${ }^{8}$. No se utiliza el drenaje al vacío de forma rutinaria. Finalmente, la piel es reaproximada con puntos subdérmicos, completando el cierre con sutura continua intradérmica de hilo absorbible sintético monofilamento de fino calibre. La herida se cubre con una cinta adhesiva porosa (intercambiada semanalmente) y, entonces, cubierta con un apósito secundario absorbente e impermeable.

Se recomienda usar cinta elástica, ajustable con velcro, firme pero cómodamente, abarcando todo el abdomen, para colaborar en la prevención de seromas y dar seguridad al paciente en la deambulación, que deberá ser irrestricta a partir del primer día posoperatorio. Se debe utilizar durante el primer mes, o extendido, a criterio del paciente. Completado ese período, se puede retornar progresivamente a la actividad física plena.

\section{El más aún}

El reajuste obtenido al final de la TSH determina la estabilidad del conjunto, en especial del 2. ${ }^{\circ}$ plano, el más importante de ellos, pues efectivamente es lo que cierra el defecto ventral y mantiene los MRA medializados (fig. 8). Para que ese resultado sea sostenido, a lo largo del tiempo, las suturas laterales impiden que la tracción sobre la línea alba neoformada pueda distenderla. Por el contrario, como se trata de una transposición, donde cada mitad del SH nace de un lado y se fija del otro, y no de simple superposición de los folletos, el anclaje es directamente proporcional a las fuerzas de separación. En esto reside la genialidad de la transposición, que también forra y protege el plan principal de reparación anteroposterior.

Los estudios histológicos del saco herniario también confirmarían su naturaleza eminentemente conjuntiva, bastante rica en fibras colágenas y revestidas de mesotelio peritoneal, confirmando el fenómeno de verdadera «aponeurotización» por la que pasa esa estructura a lo largo del tiempo, espontáneamente o incluso inducida por malla ${ }^{9-11}$. 


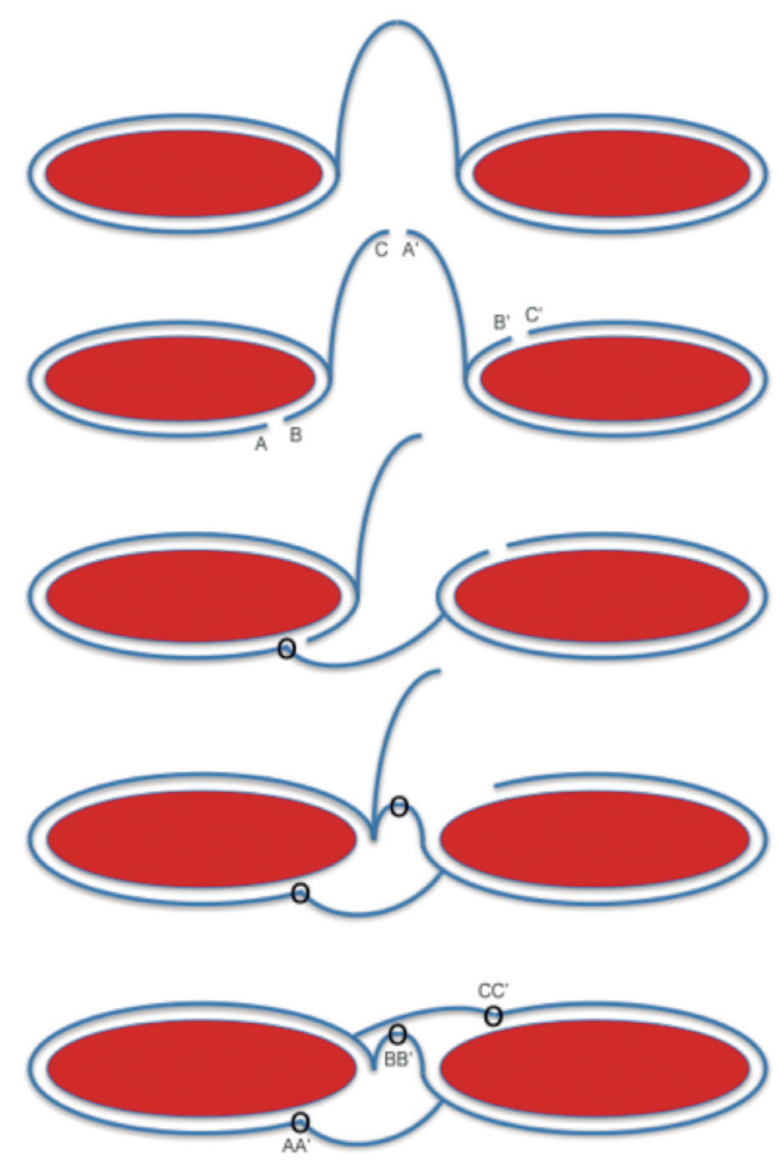

Figura 8. Representación esquemática de la transposición con el saco herniario (cortes transversales).

No ha sido rara la expresión de osificación heterotópica en el $\mathrm{SH}$ de eventraciones, de dimensiones variadas ${ }^{12}$. Estos hallazgos llamaron nuestra atención y, en una investigación aún no publicada, se tomaron muestras de SH incisional humano, que sometidas a la inmunohistoquímica, con anticuerpos monoclonales CD-133, resultaron positivas para las células madre mesenquimales en casi todos los especímenes. La conclusión que se saca es que el SH puede ser considerado banco natural de células madre, localizadas tanto en el conjuntivo o en el mesotelio de revestimiento (peritoneo parietal). Por metaplasia se forman nuevos fibroblastos que, una vez activados, estimulan los fenómenos reparativos, consolidándolos. Hecho esto, vuelven a su estado latente, menos diferenciado, hasta que sean nuevamente reclutados ${ }^{13}$.

A pesar de ello, la infección del sitio quirúrgico profundo puede acometer la integridad de los tejidos recién suturados, destruyéndolos, lo que favorecerá la dehiscencia de la herida abdominal y/o la recidiva herniaria. De la misma forma, cualquier disturbio metabólico del colágeno, sea disminuyendo su síntesis o aumentando su degradación, también podrá comprometer la longevidad de la reparación, generando nuevas recurrencias de la eventración. Desafortunadamente, hasta el momento, no se dispone de método investigativo de laboratorio que anticipe al cirujano esa deficiencia del tejido conectivo. Sin embargo, corresponde al cirujano tomar las medidas adecuadas para evitar los procesos supurativos, actuando con esmerada técnica operatoria.
Es importante recordar que los índices de recidiva, obtenidos por cirujanos en entrenamiento a largo plazo, fue del $18.75 \%$ $(15 / 80)$, en el levantamiento hecho por el autor ${ }^{4}$. En una auditoría reciente realizada en Dinamarca, de 366 operaciones para corrección sin prótesis de la hernia incisional, incluyendo procedimientos variados y realizados por cirujanos sin dedicación exclusiva a esa afección, en la cual todos los pacientes fueron seguidos cinco años completos, la tasa de fracasos fue del $17.1 \%$. Estos autores también cuestionan el real costo-beneficio del uso sistemático de la malla, en estos casos, una vez que los beneficios pretendidos (curación de la enfermedad) se equipararon a las complicaciones acumulativas y graves derivadas de su uso ${ }^{5}$. Si consideramos los peores resultados con la TSH, derivadas de las operaciones realizadas por médicos residentes, se equipararon a los de los colegas de Dinamarca citados. Si damos al cirujano general la oportunidad de perfeccionarse en la ejecución de la TSH, seguramente sus resultados tenderán a mejorar, reduciendo o espaciando las recurrencias de la enfermedad.

Merece una palabra la asociación de la TSH con las mallas. En cuanto al empaquetar la prótesis con los folletos del SH ya ha sido practicada, la experiencia que más se aproxima al procedimiento original es la de Tulloh, en Escocia, en la cual la malla fue colocada en el espacio retromuscular, de lado a lado, en 21 pacientes. Para ello, los autores suprimieron el $2 .^{\circ}$ plano de sutura, dejando el continente abdominal parcialmente abierto ${ }^{14}$. Esa es una estrategia útil, sobre todo cuando cerrar la cavidad por completo podría aumentar peligrosamente la presión intraabdominal, causando el temido síndrome compartimental. El resultado fue excelente, donde solo un paciente presentó complicaciones infecciosas en el sitio quirúrgico, que obligó a la exéresis de la prótesis, llevando a la nueva recidiva de la enfermedad.

En diciembre de 2011, iniciamos en nuestro servicio un ensayo clínico aleatorizado (triple enmascarado), en el que 62 pacientes fueron operados por un único cirujano, para corrección de hernias incisionales gigantes (defectos elípticos con al menos $10 \mathrm{~cm}$ de ancho) con la TSH. Después de completar el 3. ${ }^{\text {er }}$ plano de la reconstrucción parietal, se sorteó si el paciente recibía o no una malla de PVDF (DynaMesh-CICAT, FEG, Aachen, Alemania), como refuerzo, fijada en posición preaponeurótica. La presión de la meseta traqueal fue evaluada, en la mayoría de los casos, para monitorear aumentos significativos de la presión intraabdominal. El desenlace principal es la tasa de recidiva en cinco años y, secundariamente, las demás complicaciones de la herida y la mortalidad operatoria. En cuanto a que el seguimiento aún no se ha concluido, el análisis preliminar de tres años posoperatorios mostró no haber recidivas en ninguno de los grupos y las complicaciones locales, en general elevadas en esos pacientes, no diferían con el uso de la malla. Si, por un lado, la prótesis de PVDF no tuvo efecto deletéreo adicional en el sitio quirúrgico, su presencia parece tampoco influir en la tasa de recidiva (resultados aún no publicados). Los datos definitivos mostrarán si ella es esencial.

Ante la elevación vertiginosa de los costos con atención a la salud, a título de resarcir inversiones en nuevas tecnologías médicas (cuya eficiencia es todavía dudosa), tenemos a disposición un procedimiento viable en cualquier hospital de tamaño mediano y por cualquier cirujano con formación básica. Tal prerrogativa amplía el alcance social de esta propuesta, concebida y elaborada en una época y lugar desprovistos de recursos sofisticados. Todos estos factores fundamentan los resultados favorables de la transposición y reflejan la genialidad de sus principios orientadores. 
Sin duda alguna, debe figurar entre las opciones que el cirujano dispone, para tratar una afección que insiste en reaparecer. En caso de que esto ocurra, quedará aún la alternativa de ofrecer al paciente la reparación protésica, a pesar de las complicaciones acumulativas, algunas muy graves, relacionadas al uso de esos materiales. El médico actúa momentáneamente, pero sus acciones, buenas o malas, tienen desdoblamientos proporcionales, por lo tanto, actitudes inconsecuentes no son bienvenidas. La transposición con el saco herniario completa 50 años de vida útil, pero sus próximos cumpleaños dependerán del espíritu crítico y libre de los cirujanos, que, a ejemplo del profesor Alcino Lázaro da Silva, estén comprometidos con los desafíos presentados por la enfermedad herniaria.

\section{Bibliografía}

1. Lázaro da Silva A. Surgical correction of longitudinal median and paramedian incisional hernia. Surg Gynecol Obstet. 1979;148: $579-83$.

2. de Vries Reilingh TS, Bodegom ME, van Goor H, Hartman EHM, van der Wilt GJ, Bleichrodt RP. Autologous tissue repair of large abdominal wall defects. Br J Surg. 2007;94:791-803.

3. Rezende-Neto JB, Angarita FA, Rizoli SB, Rotstein OD. Modified triple-layer peritoneal-aponeurotic transposition: A new strategy to close the "open abdomen. J Trauma Acute Care Surg. 2015;79: 694-7.

4. Lázaro da Silva A, Vieira RG, Anjos GC. Recidiva da hérnia incisional após o tratamento pela transposição peritônio-aponeurótica longitudinal bilateral. Arq Gastroenterol. 2004;41:134-6.
5. Kokotovic D, Bisgaard T, Helgstrand F. Long-term recurrence and complications associated with elective incisional hernia repair. JAMA. 2016;316(15):1575-82.

6. Lázaro da Silva A, Ferreira AP, Ribeiro V. Estudo eletromanométrico em pacientes portadores de hérnia incisional. Rev Col Bras Cir. 1990;17(1):2-4.

7. Paulo DNS, Lázaro da Silva A. Repercussões respiratórias funcionais da hernioplastia incisional abdominal longitudinal. Rev Col Bras Cir. 1995;22:33-41.

8. Baroudi R, Ferreira CAA. Seroma: how to avoid it and how to treat it. Aesth Surg J. 1998;18(6):439-41.

9. Kather Neto JM. Sistema de fibras elásticas do saco herniário em hérnias incisionais ventrais do abdome. Estudo comparativo de seu conteúdo com o do peritônio parietal e da lâmina anterior da bainha do músculo reto do abdome [tese, Livre Docência]. Taubaté: Departamento de Medicina da Universidade de Taubaté; 1996.

10. Faria LP, Lázaro da Silva A, Rocha A. Hérnias incisionais medianas e paramedianas: estudo do saco herniário à $\mathrm{MO}$ (mesotélio, tecido conjuntivo frouxo e denso com presença de fibras colágenas, reticulares e elásticas). Rev Col Bras Cir. 1996;23(4):187-91.

11. Matapurkar BG, Bhargave A, Dawson L, Sonal B. Regeneration of abdominal wall aponeurosis: new dimension in Marlex-peritoneal sandwich repair of incisional hernia. World J Surg. 1999;23:446-51.

12. Melo RM, Mendonça ET, Mendonça EQ, Mendonça MQ. Ossificação heterotópica em saco herniário incisional - relato de caso. Rev Col Bras Cir. 2012;39(2):151-4.

13. Gotloib L, Gotloib LC, Khrizman V. The use of peritoneal mesothelium as a potential source of adult stem cells. Int J Artif Organs.. 2007;30(6):501-12.

14. Malik A, MacDonald ADH, de Beaux AC, Tulloh BR. The peritoneal flap hernioplasty for repair of large ventral and incisional hernias. Hernia. 2014;18:39-45. 